\title{
Determination of Omega-3/Omega-6 Ratio in Swine Brain Homogenate as an Animal Model for Human Nervous Tissue
}

\author{
Tero-Vescan Amelia ${ }^{1}$, Osz Bianca-Eugenia2 ${ }^{\star}$, Vari Camil-Eugen², Imre Silvia ${ }^{3}$, Hancu Gabriel ${ }^{4}$ \\ 1 Department of Pharmaceutical Biochemistry, University of Medicine and Pharmacy Tîrgu Mures, Romania \\ 2 Department of Pharmacology and Clinical Pharmacy, University of Medicine and Pharmacy Tîrgu Mures, Romania \\ 3 Department of Analytical Chemistry and Drug Analysis, University of Medicine and Pharmacy Tîrgu Mures, Romania \\ 4 Department of Pharmaceutical Chemistry, University of Medicine and Pharmacy Tîrgu Mures, Romania
}

\begin{abstract}
Objectives: The purpose of the study was to determine the omega-3/omega- 6 ratio in swine brain homogenate by HPLC with UV detection and to discuss the values obtained by comparison to the human species. Materials and methods: Determinations were performed by HPLC method using as mobile phase an isocratic mixture (A:B - 5:95) of mobile phase $A=25 \%$ acetonitrile in water and $B=$ acetonitrile with a flow-rate of $1.2 \mathrm{~mL} / \mathrm{min}$ and UV detection at 205nm. Chromatographic column: Phenomenex C8 $150 \times 4.6 \mathrm{~mm} 5 \mu \mathrm{m}$. $50 \mathrm{~g} \mathrm{swine} \mathrm{brain} \mathrm{was}$ hydrolyzed with $100 \mathrm{~mL} 0.5 \mathrm{~N} \mathrm{HCl}$, the organic phase was extracted in $50 \mathrm{~mL}$ hexane, concentrated by evaporation and resumed in $200 \mu \mathrm{L}$ acetonitrile. Results: Polyunsaturated fatty acids were separated as follows arachidonic acid (AA) - Rt $=2.69$ min, docosahexaenoic acid $(\mathrm{DHA})-\mathrm{Rt}=3.12 \mathrm{~min}$ and eicosapentaenoic acid $(\mathrm{EPA})-\mathrm{Rt}=3.97 \mathrm{~min}$. The following omega-3/omega-6 ratios were calculated (DHA + EPA)/ $\mathrm{AA}=0.572 \pm 0.451, \mathrm{EPA} / \mathrm{AA}=0.027 \pm 0.015$ and $\mathrm{DHA} / \mathrm{AA}=0.689 \pm 0.612$. Conclusions: The values obtained for these ratios should be balanced, but in reality they are in favor of the ratio denominator. Considering the physiological and nutritional similarities and that an accurate diagnosis of neurodegenerative disease is set in post-mortem, swine brain homogenate could serve as an animal model for human nervous tissue.
\end{abstract}

Keywords: HPLC-UV, eicosapentaenoic acid, docosahexaenoic acid, arachidonic acid, Omega-3 Index

Received: 13 August 2015 / Accepted: 03 September 2015

\section{Introduction}

In the past a high-fat food intake responsible for the increase of serum triglycerides and cholesterol was considered a cardiovascular risk factor, such as in the Framingham score, but nowadays the type of ingested fat is more important. Unsaturated fats are preferred to saturate and polyunsaturated fatty acids (PUFA) are considered cardioprotective factors [1].

World Health Organization (WHO) in 2009 in "Global Strategy on Diet, Physical Activity and Health" considered that a diet rich in long-chain omega-3 fatty acids (n3PUFA) could be beneficial in non-communicable disease prevention such as neurological and neurodegenerative diseases, cancer, cardiovascular disease and diabetes [2].

Literature data show that omega-3 polyunsaturated fatty acids (n-3PUFA), particularly EPA and DHA, are involved in reducing the risk of cardiovascular diseases or metabolic syndrome often a cause of neurodegenerative diseases, of Alzheimer's disease by multiple mechanisms. One such mechanism would be reducing atherogenic risk by reducing "de novo" biosynthesis of triglycerides or LDL cholesterol particle size growth [3].

It is well known that the incorporation of n-3PUFA into membrane phospholipids depends on plasma levels and therefore on food intake [4]. Determination of plasma

* Correspondence to: Bianca-Eugenia Osz

E-mail: bianca.osz@umftgm.ro levels of n-3PUFA reflects the situation in the moment of analisis (food intake in the last 4-5 hours or different causes of lipolysis) and the turn-over of free fatty acids in plasma is rapid. Omega-3 Index (n-3PUFA percentage in red blood cells (RBC) membrane phospholipids) may be a better indicator of the overall situation and is considered an independent biomarker of sudden cardiac death risk [5].

Methods of gas chromatography (GC) [6], LC/MS [7] or HPLC with UV detection [8] are described in literature to determine PUFA from biological samples.

The purpose of this study is to elaborate a HPLC-UV method to identify PUFA (EPA, DHA, AA) in swine brain homogenate and calculation ratios (EPA+DHA)/AA, EPA/ AA or DHA/AA of clinical interest.

\section{Materials and methods Standards and Reagents}

Standard substances were at least $98.0 \%$ purity and were purchased as follows EPA and DHA from Cayman Chemical Company and AA from Sigma-Aldrich.

Methanol, acetonitrile, formic acid were HPLC grade and purchased from Merck (Merck KGaA, Darmstadt, Germany).

\section{Preparation of standard solutions}

Stock solutions of $1 \mathrm{mg} / \mathrm{mL}$ in methanol were prepared and the working solution was obtained by diluting the stock with methanol. 


\section{Equipments}

AB54S (Mettler-Toledo) balance, MP225 (Mettler-Toledo) pH-meter, centrifuge 2-15 (Sigma), mixer 10 (Falc Instruments), Direct $Q$ water purification unit (Millipore), ultrasonic bath Transsonic T700H (Elma).

\section{The chromatographic system}

Biological determinations were performed on a Merck Hitachi chromatographic system consisting of: binary pump L-7100 with degasser L-7612; L-7200 automatic injector, L-7360 thermostat and DAD detector L-7455.

Chromatographic column: Phenomenex C8 150x4.6 mm $5 \mu \mathrm{m}$

The mobile phase. The mobile phase consisted of an isocratic mixture (A : B5 : 95) of mobile phase $A=25 \%$ acetonitrile in water and $\mathrm{B}=$ acetonitrile with a flow of 1.2 $\mathrm{mL} / \mathrm{min}$.

Detection was set at $205 \mathrm{~nm}$.

\section{PUFA Extraction from biological samples}

Swine brain was purchased frozen. A quantity of $50 \mathrm{~g}$ swine brain was triturated in a mortar with sea sand and left for 12 hours at $70^{\circ} \mathrm{C}$ in a water bath with $100 \mathrm{~mL}$ HCL $0.5 \mathrm{~N}$. After 12 hours a volume of $100 \mathrm{~mL}$ of $5 \% \mathrm{NaCl}$ was added to reduce the acidity of the sample (dilution with increased ionic strength). The organic phase containing the compounds of interest was extracted in $50 \mathrm{~mL}$ hexane, concentrated by evaporation, resumed in $200 \mu \mathrm{L}$ of acetonitrile and injected into the chromatographic system.

\section{Results}

Previously, the optimised separation and detection were investigated. The wavelength for analysis was chosen based on the maximum absorption of the three fatty acids at 205 $\mathrm{nm}$ (Figure 1). The method permitted a good separation under reversed phase conditions of the three PUFA: AA, DHA and EPA (Figure 1, detail).

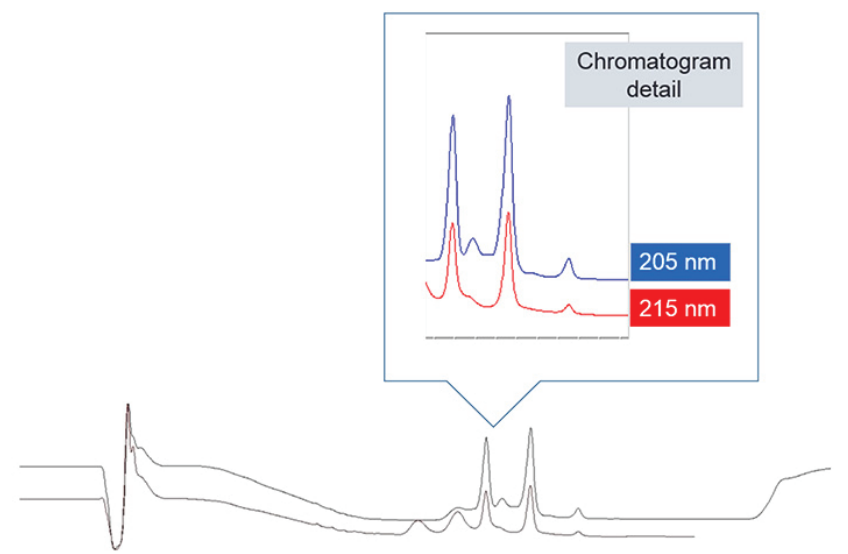

Fig. 1. The chromatograms of the standard mixture of PUFA with a concentration of $10 \mu \mathrm{g} / \mathrm{mL}$ each acid, at $205 \mathrm{~nm}$ and $215 \mathrm{~nm}$, respectively
Chromatogram of swine brain homogenate is shown in Figure 2.

A ballanced ratio n-3PUFA/n-6PUFA provide neuroprotection, while in reality this ratio is slightly unbalanced in favor of n-6PUFA. The values of this ratio are presented in Table I.

Table I. Relationship between different levels of PUFA in the brain homogenate

\begin{tabular}{lc}
\hline Ratio & Average value $(\mathrm{n}=3$ samples $) \pm$ SD \\
\hline EPA/AA & $0.027 \pm 0.015$ \\
DHA/AA & $0.689 \pm 0.612$ \\
EPA+DHA/AA & $0.572 \pm 0.451$ \\
\hline
\end{tabular}

\section{Discussions}

The results presented in Table I show a n-3PUFA/n-6PUFA subunitary ratio. In humans, this ratio is probably even more unbalanced considering that swine food contains fishmeal among other things, with increased content of n3PUFA [9].

The classic HPLC with UV detection method, for the determination of PUFA without derivatization is limited by the large quantity of utilizied brain samples. Using swine brain homogenate can draw conclusions on the n3PUFA/n-6PUFA ratio, the results can be extrapolated to humans if we consider similar, omnivorous diets, rich in saturated and monounsaturated fats and low in PUFA of the two species and that human studies are not possible because of ethical considerations [10].

Recent studies show that the similarities between the two species are not limited to nutrition but also to the gastrointestinal tract anatomy, neuroanatomy, body composition and cardiovascular risk factors: high LDL-cholesterol and low HDL cholesterol, obesity, metabolic syndrome [11] and Omega-3 Index [12].

DHA is involved in developing of fetal nervous system and retina [1]. Also, after subcutaneous administration in

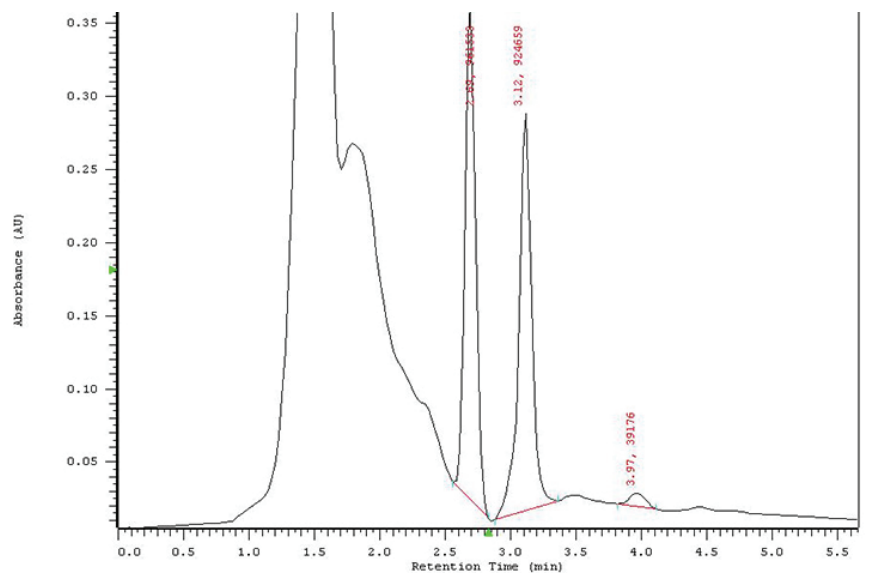

Fig. 2. Chromatogram of the swine brain homogenate 
rats, DHA increases seizure latency in a neuronal excitability model caused by pentetrazol [13].

Recent studies show a possible antipsychotic effect of n-3PUFA. A study comparing antipsychotic effects of n3PUFA (in a model of psychosis induced by administration of apomorphine, a $\mathrm{D}_{2}$-dopamine agonist) with chlorpromazine in rats show that a possible antipsychotic effect of $n$-3PUFA could be due to the reduction of oxidative stress and the influence on the plasma levels of malondialdehyde and glutathione [14].

Another study shows that EPA and DHA present similar antidepressants effects of $17 \beta$-estradiol by regulating serotonergic neurotransmission and decreasing the synthesis of inflammatory cytokines [15].

High intake of n-3PUFA influences the insulin/glucagon ratio modified in type 2 diabetes, often a cause of neurodegenerative diseases and cognitive impairments, while, generally, high blood sugar levels are positively associated with risk of Alzheimer's disease [16].

Another study performed on Streptozotocin induced diabetic rats shows that additional intake of $n-3 P U F A$ improves exploratory behavior and memory and prevents secondary neurosensorial impairments caused by diabetes [17].

The role of the n-3PUFA and especially of DHA in the treatment of spinal cord injury complications is based on reduction of excitotoxicity, inflammation, oxidative stress and lipid peroxidation. DHA increases neuronal survival by unknown mechanisms after spinal cord injury. A possible mechanism would be reducing glutamate toxicity, which is released in large quantities especially after traumatic spinal cord injury [18].

Another important effect of n-3PUFA is the transformation by cyclooxygenase (COX)-2 and 5-lipoxygenase pathway into resolvin, respectively resolvin series $\mathrm{E}$ having EPA as precursor and resolvin series D with DHA as precursor. Resolvin decrease neutrophil migration into inflamed tissues, promotes phagocytosis of apoptotic cells and decrease inflammatory cytokine release [19].

Neuroprotectins have similar effects to resolvins, but are localized in the brain; literature data show neuroprotective effects and reduce of neuropathic pain in animal models of stroke and Alzheimer's disease [20].

The content of DHA in the brain is increased. It is found mainly as a component of phospholipids and smaller quantities in the composition of sphingomyelin and ceramide. DHA is released from membrane phospholipids by the catalysis of phospholipase $A_{2}$, it is then hydroxylated by 15-lipoxygenase-1 with the formation neuroprotectin $\mathrm{D}_{1}$. Studies show that neuroprotectin $\mathrm{D}_{1}$ reduces $\beta$-amyloid precursor protein converting into 42 amino acid amyloidbeta, reducing therefore characteristic neurodegeneration in Alzheimer's disease [20].

\section{Conclusion}

Based on the physiological similarities, in terms of food intake, values of cardiovascular risk factors LDL/total cholesterol ratio, Omega-3 Index or omega-3/omega-6 ratio and considering ethics regarding the diagnosis of neurodegenerative diseases in human (absence of biochemical biomarkers and presumptive clinical diagnosis) swine could be a highly accessible animal model. Values obtained for the omega-3/omega-6 ratio in swine brain homogenate show that it is unbalanced in favor of the denominator, EPA and DHA deficiency in the brain could be an additional independent risk factor in cognitive impairments.

\section{Acknowledgment}

This paper was published under the frame of European Social Found, Human Resources Development Operational Program 2007-2013, project no. POSDRU/159/1.5/S/136893

\section{References}

1. Gil A, Gil F. Fish, a Mediterranean source of n-3 PUFA: benefits do not justify limiting consumption. Br J Nutr. 2015;Suppl2:S58-67.

2. World Health Organization. Interventions on diet and physical activity: what works: summary report, 2009, available at: http://apps.who.int/iris/ handle/10665/44140.

3. Huhn S, Kharabian Masouleh S, Stumvoll M et al. Components of a Mediterranean diet and their impact on cognitive functions in aging. Front Aging Neurosci. 2015;7:132.

4. Wu JH, Lemaitre RN, King IB et al. Association of Plasma Phospholipid Long-Chain Omega-3 Fatty Acids with Incident Atrial Fibrillation in Older Adults: The Cardiovascular Health Study. Circulation. 2012;125(9):10841093.

5. von Schacky C. Cardiovascular disease prevention and treatment. Prostaglandins Leukot Essent Fatty Acids. 2009;81(2-3):193-198.

6. Onozato $\mathrm{M}$, Nishikiori $\mathrm{M}$, lizuka $\mathrm{H}$ et al. Determination of sex-based differences in serum $\gamma$-linoleic acid and dihomo- $\gamma$-linoleic acid using gas chromatography-mass spectrometry. J Chromatogr B Analyt Technol Biomed Life Sci. 2015;997:116-21.

7. Aslan M, Özcan F, Aslan I, Yücel G. LC-MS/MS analysis of plasma polyunsaturated fatty acids in type 2 diabetic patients after insulin analog initiation therapy. Lipids Health Dis. 2013;12:169.

8. Guarrasi V, Mangione MR, Sanfratello $V$ et al. Quantification of underivatized fatty acids from vegetable oils by HPLC with UV detection. J Chromatogr Sci. 2010;48(8):663-668.

9. Su YB, Bi XH, Ma XK, et al. Determination and prediction of the digestible and metabolizable energy content of lipid sources fed to growing pigs. Animal Feed Science and Technology, 2015; http://dx.doi.org/10.1016/j. anifeedsci.2015.07.004

10. Miller ER, Ullrey DE. The pig as a model for human nutrition. Annu Rev Nutr. 1987;7:361-382.

11. Koopmans SJ, Schuurman T. Considerations on pig models for appetite, metabolic syndrome and obese type 2 diabetes: From food intake to metabolic disease. Eur J Pharmacol. 2015;759:231-239.

12. Whelan J, Gouffon J, Zhao Y. Effects of dietary stearidonic acid on biomarkers of lipid metabolism. J Nutr. 2012;142(3):630S-634S.

13. Trépanier MO, Kwong KM, Domenichiello AF et al. Intravenous infusion of docosahexaenoic acid increases serum concentrations in a dosedependent manner and increases seizure latency in the maximal PTZ model. Epilepsy Behav. 2015;50:71-76.

14. Kokacya MH, Inanir S, Copoglu US et al. The Antipsychotic Effects of Omega-3 Fatty Acids in Rats. Am J Med Sci. 2015 Jul 21. [Epub ahead 
of print].

15. Jin Y, Park Y. n-3 polyunsaturated fatty acids and $17 \beta$-estradiol injection induce antidepressant-like effects through regulation of serotonergic neurotransmission in ovariectomized rats. J Nutr Biochem. 2015; pii: S0955-2863(15)00104-7 [Epub ahead of print].

16. Kerti L, Witte AV, Winkler A, et al. Higher glucose levels associated with lower memory and reduced hippocampal microstructure. Neurology. 2013;81:1746-1752.

17. Yadav S, Mitha KV, Shenoy MT, et al. Beneficial effect of Omega-3 polyunsaturated fatty acids on neurosensorial impairments and oxidative status in Streptozotocin induced diabetic rats. Indian J Physiol Pharmacol.
2014; 58(4):346-353

18. Huang WL, King VR, Curran OE, et al. A combination of intravenous and dietary docosahexaenoic acid significantly improves outcome after spinal cord injury. Brain. 2007;130(Pt 11):3004-3019.

19. Gilbert K, Bernier J, Godbout R, Rousseau G. Resolvin D1, a metabolite of omega-3 polyunsaturated fatty acid, decreases post-myocardial infarct depression. Mar Drugs. 2014; 2(11):5396-5407.

20. Stark DT, Bazan NG. Neuroprotectin D1 induces neuronal survival and downregulation of amyloidogenic processing in Alzheimer's disease cellular models. Mol Neurobiol. 2011; 3(2):131-138. 